\title{
Acknowledgment of Ad Hoc Reviewers
}

The Editor gratefully acknowledges the following individuals who reviewed manuscripts submitted to the journal Cultural Diversity \& Ethnic Minority Psychology in the past year.

Saida M. Abdi

Sawssan Ahmed

Riana Elyse Anderson

Arthur Andrews III

Consuelo Arbona

Eunice Moraa Areba

Shaljan Areepattamannil

Adria Armbrister

Astraea Augsberger

Ozlem Ayduk

Guadalupe Bacio

Joaquín Bahamondes

Meeta Banerjee

Sheri Bauman

Adam Beaupre

Frederick Beauvais

Angela R. Bethea-Walsh

Nida Bikmen

Kevin R. Binning

Magdalena Bobowik

Luisa N. Borrell

Guy Boysen

Jasmin Brooks*

NiCole T. Buchanan

Sara Buckingham

Jason Burrow-Sanchez

Mia Smith Bynum

Latifat Cabirou*

Howard Cabral

Daisy Camacho-Thompson

Sierra Carter

Kimberly Chaney

Tzu-Fen Chang

Linda Charmaraman

Nitasha Chaudhary

Nayeli Y. Chavez-Dueñas

Jacqueline Chen

Danhong Chen

Chi-Ying Cheng

Alice Cheng

Yuen Mi Cheon

Dorothy Chin

Becky Choma

Joyce Chu

Sauro Civitillo

Erica E. Coates*

Kevin Cokley

Stephen Colmant

Jeff Cookston

Theodore V. Cooper

Rebecca Covarrubias

Maureen Craig

Annette Crisanti

Annamaria Csizmadia

Carolyn Cutrona
Felix Danbold

Layla Dang*

Anthony De Jesus

Charlotte De Kock

Wendy de los Reyes Moore*

Sofie de Smet

Melanie M. Domenech

Rodriguez

Richard Douglass

Emma Elliott-Groves

Christina Engelken*

Qijuan Fang

Yaqoot Fatima

Laura Ferrer-Wreder

Gillian Finchilescu

Jillian Fish

Sycarah Fisher

Melissa Ann Flores

Diana Formoso

Marisa Franco

Sheila Frankfurt

Lauren Freeman

Cynthia M. Frisby

Marta Fülöp

Julie Gameon*

Glenn Gamst

Danielle Gaucher

Anisa Goforth

Diane Carol Gooding

Bernard Gorman

Josefina Grau

Nyasha Grayman-Simpson

Tabitha Grier-Reed

Lusine K. Grigoryan

Qurat-ul-ain Gulamhussein

Mignonne C. C. Guy

Lindsay S. Ham

Elissa Hamlat

Jill V. Hamm

Catherine E. Harnois

Jake Harwood

Erin Hennes

Julia Meredith Hess

Kristina Hood

Meredith Hope

Elan C. Hope

James P. Huguley

Carla D. Hunter

Masumi Iida

Derek Iwamoto

Aaron P. Jackson

Valeria Jackson
Farrah Jacquez

Roshni Janakiraman*

Rich Janzen

Elizabeth Jelsma*

Marc P. Johnston-Guerrero

Thomas Joiner

Francine Karmali

Ummul Kathawalla

Jill Keith

Hali Kil

Isok Kim

Joanna Kim

Jung-In Kim

Bonnie Klimes-Dougan

George Knight

Mary Kate Koch

Philip Kreniske

Ethan Kross*

Alex Kulick

Jonas Kunst

Jenny Kurman

Keren Ladin

Amanda Lahiere*

Giselle Laiduc*

Richard Lalonde

Antoinette M. Landor

Tonisha Lane

Sandraluz Lara-Cinisomo

Anna S. Lau

Daniel B. Lee

Matthew Lee

Frederick Leong

Jioni A. Lewis

Luye $\mathrm{Li}$

Lisa Libby

Susana Ayala Lopez

Beatriz Macias

Juan M. Madera

Ijeoma Madubata*

Satia Marotta*

David Marx

Isidro Maya-Jariego

Silvia Mayen*

Elizabeth McConnell

Mallory McCord

Laurie McCubbin

Craig McGarty

Kyle McLean

Sharde' McNeil Smith

Ellen Hawley McWhirter

Marisa Mealy

Alan Meca

Yara Mekawi

Sonia Mendoza

Laurie Meschke
Lauren Mims

Yuko Mizuno

Ursula Moffitt

Kristine Molina

Sherry Davis Molock

Ui Jeong Moon

Karen Moran Jackson

Oswaldo Moreno

Sydney K. Morgan

Hector Myers

Misaki N. Natsuaki

Andy Ng

Caroline Ng Tseung-Wong

Diem Julie Nguyen

Angela-MinhTu D. Nguyen

Jacqueline Nguyen

Laurie O'Brien

Mary-Frances O'Connor

Joan Ostrove

Robert Outten

Gina Owens

Yingqiu Pan

Irene J. K. Park

Hong-Jae Park

Carlton W. Parks

Michael Perez*

Jessica Perrotte*

Bridgette Peteet

Savannah Pham*

L. Taylor Phillips

Evava Pietri

Delishia Markayle Pittman

Ashby Plant

Michael Platow

Scott Wayne Plunkett

Matthew Quesnel

Nadia Rania

Luke J. Rapa

Nils Karl Reimer

Jessica D. Remedios

Shuzheng Ren

Tuuli Anna Renvik

Maryse Richards

Debra Roberts

Leoandra Onnie Rogers

Barry Rosenfeld

Lisa Rosenthal

Floyd W. Rudmin

Nancy Rumbaugh Whitesell

Temilola Salami

Diana T. Sanchez

Jun Sasaki 
Anne Saw

Maja Schachner

Deborah Schooler

Miriam Schwarzenthal

Christie Scollon

Lalaine Sevillano

Kelley Sittner

Allison Louise Skinner

Leann V. Smith

Chauncey Smith

Nadia Sorkhabi

Angelitta Spells

Adrian Stanciu

Gabriela Livas Stein

Abigail J. Stewart

*Denotes a co-reviewer
Steve Stone

Hanna Suh

Marie-Anne Suizzo

Terri Sullivan

Grace Sumabat Estrada

Moin Syed

Tamara Taggart

Karen Tao

Chelsie Temmen*

Courtney S. Thomas Tobin

Nathan Todd

Jessica Totsky*

Noriko Toyokawa

Pratyusha Tummala-Narra
Adriana Umaña-Taylor

Ibette Valle*

Kali S. Van Campen

Yolanda Vasquez-Salgado

Alexandra Vázquez

Efren Velazquez

Jana Vietze

Vanessa Volpe

Sophie Deborah Walsh

Richard Wampler

Monnica Williams

Adriana Wilson*

Leigh Wilton
Melissa Witkow

Audrey Wittrup*

Gloria Wong-Padoongpatt

Laura Wray-Lake

Jueyu Wu

Qiaobing $\mathrm{Wu}$

Asli Cennet Yalim

Miwa Yasui

Tiffany Yip

Luis H. Zayas

Katharine H. Zeiders

Xiang Zhou

Linda Zou 